\title{
GENEALOGÍA DE LA INVESTIGACIÓN EN LA FACULTAD DE EDUCACIÓN DE LA UNIVERSIDAD SURCOLOMBIANA
}

\author{
Nelson Ernesto López Jiménez \\ María Fernanda Pérez Gutiérrez** \\ Wilson Perdomo Cortés***
}

\section{RESUMEN}

El presente artículo muestra los resultados de la investigación "Hacia la Genealogía de la Investigación en la Facultad de Educación de la Universidad Surcolombiana", que pretende explicar los momentos y circunstancias de las investigaciones a partir de las contradicciones e intereses que se manifiestan y caracterizan en un período histórico en particular de la universidad. En atención a ello, el equipo investigador del Grupo de Investigación PACA, desarrolló una revisión sistemática de los informes y documentos de las investigaciones realizadas en el periodo comprendido entre 1970 al 2010. De acuerdo con lo anterior, se procedió al análisis de cada una de las décadas para comprender los sucesos y contextos que generaron las investigaciones de la Facultad de Educación.

PALABRAS CLAVE: genealogía, interés, causas, motivaciones, impactos.

\section{JUSTIFICACIÓN}

La Universidad Surcolombiana, cuenta en la actualidad con siete (7) Facultades entre las cuales se destaca la Facultad de Educación como pionera de muchos de los programas, proyectos y procesos que le han dado existencia y reconocimiento a la Universidad; también ha servido de referente de muchos proyectos de investigación que la han convertido en objeto de estudio y la han considerado referente sustantivo cuando de excelencia y calidad se hace mención.

En este contexto, el desarrollo de la investigación sobre "La genealogía de la investigación en la Facultad de Educación" realizó un análisis riguroso y coherente en relación con la historia y la realidad actual de la Facultad de Educación en materia de investigación.

La genealogía es una técnica histórica en la que se cuestiona el surgimiento comúnmente entendido de diversas creencias filosóficas y sociales, mostrando historias alternativas y subversivas de su desarrollo; la genealogía no se pregunta por el origen de las ideas de los valores o de las identidades sociales sino que muestra cómo éstas emergen producto de las relaciones de fuerza (Nietzsche). Por lo anterior, se intentó en esta investigación rastrear de una manera sistemática las razones y circunstancias por las cuales se ha investigado en la Facultad de Educación de la Universidad Surcolombiana.

La investigación, inició con la elaboración y construcción de un diagnóstico sistemático con el fin de vislumbrar una radiografía inicial de la genealogía de la investigación. El presente estudio permitió ubicar en el tiempo y en el espacio las formas, los enfoques epistémicos, los métodos, los actores, los programas, las líneas de investigación y las

* Ph.D en Ciencias Sociales, Niñez y Juventud. Red CLACSO. Septiembre de 2012.

** Estudiante de Maestría en Educación, Área de Profundización Docencia e Investigación Universitaria.

***Comunicador Social y Periodista. Universidad Surcolombiana. 
acciones que han permitido la concreción de la investigación en la Facultad de Educación como un eje central de su desarrollo.

\section{REFERENTE LEGAL}

Se consideró pertinente en el proyecto de investigación, realizar una aproximación inicial de las políticas en materia de investigación en relación con el contexto mundial, nacional e institucional que permitiera comprender desde el enfoque genealógico.

Acerca de la política de investigación a nivel internacional.

De acuerdo con la Conferencia Mundial sobre la Educación Superior que se realizó en el año 1998 en la ciudad de París, se reunieron 182 estados con el fin de discutir sobre la problemática de la educación superior con miras a proponer estrategias que oriente el tipo de educación superior que se espera para el próximo siglo.

En la Declaración de la Conferencia Mundial se menciona: como la sociedad está cada vez más basada en el saber [...], la educación superior y la investigación son en la actualidad los componentes esenciales del desarrollo cultural, socioeconómico y ecológicamente viable de los individuos, las comunidades y las naciones". Así pues, el desarrollo de la educación superior debe figurar entre las más altas prioridades nacionales.

Es importante mencionar que una de las funciones de la educación superior que debatieron en la Conferencia Mundial fue la investigación como tema principal, ya que es una función elemental en la educación superior porque fortalece la formación de los estudiantes a partir de principios, procesos, fundamentos y prácticas académicas importantes para desempeñarse como profesional o investigador, que busca innovar, transformar procesos y solucionar la problemática del contexto.

En ese sentido, en el marco de la Conferencia Mundial en París sobre la Educación Superior-2009 denominada "la nueva dinámica de la educación Superior y la investigación para el cambio y el desarrollo", retoma los argumentos y conclusiones de las conferencias realizadas en Cartagena de Indias, Macao, Dakar, Nueva Delhi, Bucarest y El Cairo que brinda elementos sustanciales para el desarrollo de la educación superior en el mundo.

En lo que concierne al aspecto de la investigación y la innovación se señala la importancia de incrementar la financiación en investigación y desarrollo para la creación de iniciativas y estrategias que vinculen el sector público y privado en pequeñas y medianas empresas. Según el informe sobre la educación superior en América Latina y el Caribe 2000-2005 indica que el presupuesto en Latinoamérica para educación superior es inferior al 1,5\% del PIB, lo cual es evidentemente insuficiente para atender las necesidades de un sistema en desarrollo y, particularmente, las exigencias en materia de investigación. Por lo tanto, las inversiones que se hacen a las instituciones de educación superior son mínimas para el desarrollo de la ciencia y la tecnología. Es preciso mencionar que la investigación está directamente concentrada en las universidades y centros públicos -estatales, la carencia del gasto público influye en la capacidad de producción científica de los países latinoamericanos.

La Dirección de Ciencia, Tecnología e Industria de la OCDE señala que la política en materia de educación superior se ha centrado en aspectos relacionados con los resultados de la enseñanza y el aprendizaje, la calidad y la financiación de las instituciones. 
Por su parte, la política de investigación ha abordado cuestiones como las políticas y las normas que los países aplican para desarrollar y orientar la investigación y su base de conocimientos. Cada una de ellas se ha concentrado en los parámetros y lineamientos de funcionamiento de las instituciones de la educación superior, se espera que la investigación, la educación y la innovación se integren a las necesidades de la sociedad.

\section{Acerca de la política de Investigación a Nivel Nacional}

En el marco de la investigación en Colombia, se ha generado cambios en la estructuración de su política, tomando como referencia el documento 2019 Visión Colombia II Centenario agenciada por la Presidencia de la Republica, el Departamento Nacional de Planeación y Colciencias lideraron la propuesta para consolidar el crecimiento y el desarrollo social de la ciencia, la tecnología y la innovación. De esta manera el documento presenta de manera sucinta los momentos históricos que incidieron en el desarrollo de la ciencia, tecnología e innovación en Colombia, soportado en los referentes legales que apoyaron a la estructuración de la política en materia de investigación.

En el año 1968 se creó Colciencias, como entidad de financiamiento para la producción del conocimiento científico, que propició diferentes programas y proyectos de carácter tecnológico que permitió la creación de estudios de posgrados con instituciones de educación superior en el mundo, que brindaron las garantías fundamentales en el proceso de formación de docentes e investigadores. Es pertinente señalar que el proceso de formación fue de vital importancia para los grupos de investigación, sus investigaciones se consideraron como actividad individual de cara al reconocimiento institucional de la educación superior.

En la década de 1980, el desarrollo institucional se caracterizó básicamente por introducir enfoques, metodologías de gestión y negociación de tecnología incorporadas a plantas, equipos y procesos en el sector productivo. Como resultados de estos procesos, los grupos de investigación de las universidades públicas se hicieron más visibles en el contexto nacional, además la Universidad Nacional inició la creación de programas doctorales.

En la década del 1990, la Ley de Ciencia y Tecnología constituyó el Sistema Nacional de Ciencia y Tecnología creado mediante el Decreto-Ley 585 de febrero 1991, que definió los once programas nacionales de Ciencias y Tecnología (Biotecnología, Ciencias Básicas, Ciencias Sociales y Humanas, Ciencias del Mar, Ciencias del Medio Ambiente y del Hábitat, Estudios Científicos de la Educación, Ciencias de la Salud, Ciencias de la Electrónica, Telecomunicaciones e Informática, Ciencias de la Energía y Minería, Ciencias Agropecuarias y el Programa de Desarrollo Tecnológico Industrial y Calidad) para atender las necesidades científicas de cada uno de los Grupos de Investigación. En este periodo, la investigación era débil e incipiente debido a que en Colombia, los recursos que invertían a ciencia y tecnología eran mínimos y la creación de doctorados y formación de docentes en el campo de la investigación era un número minoritario.

En ese sentido Colciencias avanzó en la consolidación de las Comisiones Departamentales de Ciencia y Tecnología, con el apoyo financiero y técnico de las regiones para la generación del conocimiento. Colciencias lideró los once programas nacionales vinculados a las seis áreas de conocimiento (investigación, materia y energía, Procesos Bioló-

+ La Genealogía se entiende como el estudio y seguimiento del recorrido histórico de la investigación en la Facultad de Educación, que permita identificar y caracterizar los
enfoques epistemológicos, los métodos y formas de investigación, las líneas de investigación, los actores de la investigación, los resultados o impactos de la investigación, enfoques epistemológicos, los métodos y formas de investigación, las líneas de investigación, los actores de la investigación, los resultados o impactos de la investigación,
durante el tiempo de existencia de la Facultad. 
gicos, Agroalimentarios y Biodiversidad, Ser Humano y su Entorno Educación, Cultura e Instituciones y Gestión del Conocimiento, Aplicaciones Sociales y Convergencia Tecnológica), con este auge Colciencias promueve una propuesta nacional para el desarrollo de capacidades científicas, tecnológicas y de innovación que generó impacto en las regiones de producción del conocimiento.

A partir de la historia de la investigación en Colombia se puede evidenciar una gran necesidad de la producción del conocimiento en relación con el desarrollo económico, social y ambiental del país, lo cual se direcciona de manera desarticulada en las áreas del conocimiento. La inversión que se hace a la ciencia y la tecnología no responde a las necesidades reales que a academia y la investigación exige, como por ejemplo la formación de vértice en el campo educativo, así como, el manejo de recursos óptimos que garanticen calidad, rigurosidad y validez en los análisis y resultados investigativos.

\section{Acerca de la Política de Investigación de la Universidad Surcolombiana}

Es necesario retomar los referentes legales que aportaron en la consolidación de la política de investigación de la universidad Surcolombiana. En la década del 70, 80 y 90 surgieron tres momentos importantes como fue la creación de CESCO, CIDEC Y DIGEIN que orientaron los lineamientos básicos de la investigación y producción del conocimiento del departamento del Huila.

La Universidad Surcolombiana, inicia una gran trayectoria en el campo de la ciencia y la tecnología, con grandes aciertos y desaciertos, nace el CENTRO DE ESTUDIOS SURCOLOMBIANOS (CESCO) como primera experiencia investigativa, creado por el Rector del Instituto Técnico Surcolombiano - ITUSCO - Doctor Marco Fidel Rocha en 1971. El objetivo de CESCO, fue estudiar los problemas regionales y nacionales que afectaban el desarrollo integral de la comunidad.

Sin embargo, CESCO no figuraba en el organigrama de la Universidad, ni contaba con infraestructura, razón por la cual, los estudiantes del programa de Lingüística y Literatura presentaron ante el Consejo Académico Universitario una propuesta de reestructuración donde planteaban la importancia de obtener presupuestos para el fomento de la investigación, así como también brindar asesorías de tesis y monografías. A partir de 1984 CESCO se reestructura y se denomina CIDEC (Centro de Investigaciones y Desarrollo Científico), donde surge la propuesta original del estatuto.

DIGEIN afianzó el trabajo ampliando a varios ejes de estudios las diferentes investigaciones que en él se desarrollaban, después de los avances y desarrollos en la Universidad Surcolombiana, el Sistema de Investigaciones se institucionaliza mediante el Acuerdo $\mathrm{N}^{\circ} 064$ de 2001 Estatuto de Investigaciones de la Universidad Surcolombiana el cual se derogó con el Acuerdo № 013 de 2005.

El Estatuto de Investigaciones y el Sistema de Investigación de la Universidad Surcolombiana brindan el soporte normativo e institucional (objetivos, estrategias y principios del Sistema de Investigación, políticas de investigación, estructura del Sistema de Investigaciones, criterios y trámites para la presentación de proyectos de investigación, recursos financieros para el Sistema de Investigación y estímulos)que fortalece los procesos académicos e investigativos de la Universidad, con el Estatuto de Investigaciones se creó la Vicerrectoría de Investigación y Proyección Social, como organismo dinamizador de procesos investigativos vinculados a las áreas del conocimiento que impulsó a 
los Grupos de Investigación la categorización a Colciencias.

Además, la Universidad estableció la cadena formativa en investigación (ONDAS, semilleros de Investigación, jóvenes investigadores y Grupos de Investigación), como centro de la actividad investigativa que se desarrolla dentro de la universidad, ha brindado un respaldo a las labores docentes toda vez que se encaminan al desarrollo de la calidad académica; no obstante, en el año 2005 se presentaron falencias como la poca investigación docente, la desmotivación y desinterés de docentes hacia la investigación, así mismo, no existía precisión en políticas, áreas y líneas de investigación.

De otra parte, la poca preparación de los docentes en metodología y técnicas de investigación llevaron a que la investigación en la Universidad se diera de manera paulatina lo cual se logró contrarrestar con una asignación adecuada del tiempo ejercido por los profesores, con recursos propios que permitieron ejecutar diferentes proyectos investigación.

\section{METODOLOGÍA DE LA INVESTIGACIÓN:}

La investigación sobre la genealogía de la investigación en la Facultad de Educación parte de una revisión sistemática de los informes de investigación realizados en el periodo comprendido de 1970-2010, revisión que permitió estructurar una estrategia básica que indagaba por las razones, las circunstancias, los intereses y los porqué se realizaron las investigaciones indagadas. La investigación "se asume como un proceso que se alimenta continuamente, de y en la confrontación permanente de las realidades intersubjetivas que emergen a través de la interacción del investigador con los actores de los proceso y realidades socio-culturales y personales objeto de investigación así como del análisis de la documentación teórica, pertinente y disponible" (Sandoval, 1996).

El equipo investigador tuvo acceso a fuentes primarias, con el fin de recuperar la voz de los actores, e interactuar con los docentes líderes de las investigaciones desarrolladas, lo cual permitió la consecución de elementos esenciales que hacen parte de la historia, del contexto y de la realidad que integran el corpus teórico de la genealogía.

\section{Instrumentos de Investigación}

De acuerdo con la naturaleza de la investigación se definieron los siguientes instrumentos: Revisión Documental: Se realizó una revisión detallada de los informes de investigación de las décadas de 1970 al 2010 de la Facultad de Educación.

Análisis documental: Se revisó de manera sistemática los documentos oficiales e informes finales de los proyectos de investigación que se desarrollaron en cada una de las décadas, a partir del año 1970 al 2010.

Video testimonial: Es el elemento sustantivo para el logro de los objetivos de la investigación, lo cual recoge los testimonios directos de las audiencias que de manera voluntaria aceptaron participar en el desarrollo de la investigación.

Seminario - Taller: Se desarrolló un taller dirigido a los docentes que participaron en las investigaciones de la Facultad de Educación, con el Seminario Taller se conocieron las diferentes percepciones, experiencias de las investigaciones que generaron impacto en la región Surcolombiana además con este trabajo aporto elementos de análisis que 
enriqueció la presente investigación.

\section{RESULTADOS:}

La Facultad de Educación fue pionera de muchos proyectos de investigación de carácter institucional e individual. En algunos documentos se menciona el desarrollo de diferentes investigaciones como, el Atlas Lingüístico del Huila, la Reestructuración del Centro de Estudios Surcolombianos (CESCO), el Análisis Ocupacional del Administrador Educativo, las Causas Sociales de los Problemas de Salud en Rivera, la Asistencia Técnico Deportiva a las Juntas Municipales de Deportes y el Programa de Integración Universitaria para la Docencia y la Investigación del Español Americano. No obstante, el equipo investigador encontró los informes de investigación del Atlas Lingüístico y Reestructuración del Centro de Estudios Surcolombianos (CESCO) que reposan en Archivo Central y en la Biblioteca, de los otros que se mencionaron no se encontró ningún tipo de informe $o$ anteproyecto.

El proyecto Atlas Lingüístico surgió a partir del Trabajo de Grado que desarrolló el profesor Luis Humberto Alvarado relacionado con el aspecto fonológico de la comunidad guambiana, este trabajo aportó aspectos esenciales en la elaboración de la propuesta, además en los cursos de fonética y fonología que ofrecía el programa de Literatura fue un factor fundamental para que desarrollara el trabajo de investigación y graduara a la primera promoción de estudiantes del programa de Literatura.

Es preciso señalar que el proyecto de investigación se desarrolló como un requisito de grado - monografía de los estudiantes. Esta investigación cerró su ciclo y no continuo en la línea de investigación de la Facultad de Educación. El informe final terminó en la primera etapa y publicó el primer volumen de la documentación bibliográfica. Este proyecto fue financiado por Colciencias.

El equipo de docentes del programa de Licenciatura en Lingüística y Literatura apoyó diferentes investigaciones para consolidar la cultura investigativa de la Universidad Surcolombiana que permitiera el reconocimiento de sus experiencias investigativas con otras instituciones de educación superior.

En ese sentido se presentó un anteproyecto sobre la reestructuración del Centro de Estudios Surcolombianos (CESCO) en el año 1978, cuyo propósito estaba orientado a reestructurar el CESCO por medio de Resolución Orgánica de la Rectoría de la Universidad Surcolombiana, en la que se modificara y ampliara los estatutos, la metodología y los programas. El proyecto se gestó a partir de las necesidades de muchos docentes y estudiantes del programa de Licenciatura en lingüística y Literatura que en esa época desarrollaban diferentes investigaciones; por lo tanto, sus trabajos no contaban con recursos financieros para suplir los gastos del trabajo de campo, elaboración de informes y viáticos, razón por la cual los investigadores prefirieron consolidar los desarrollos investigativos del Centro para proyecciones futuras a nivel regional, nacional e internacional que propició vínculos con otras instituciones de educación superior.

Es conveniente señalar que esta iniciativa de investigación se consideró como un proyecto institucional que buscó fortalecer la dinámica del Centro y el desarrollo de investigaciones futuras de la comunidad universitaria. 
En la década de 1980 surgieron diversos proyectos de investigación que generaron impacto en la región surcolombiana y en el sector educativo. En el año 1984 se presentó el informe final de la investigación Perfil Teórico del Docente para el Departamento del Huila, que fue liderado por los docentes Diana Patricia Cristancho, Julian Colunge, Disnarda Mora, Antonio Iriarte Cadena, Hilda Bohórquez de Valderrama y Daniel Yovanovich. Este trabajo se desarrolló en dos fases.

La primera fase consistió en un trabajo teórico que retomó aspectos fundamentales del contexto histórico, social e ideológico. La investigación surgió de la necesidad de conocer históricamente los desarrollos que ha tenido la práctica pedagógica del docente a lo largo de los años y la relación existente con el proceso de formación.

Respecto a la segunda fase de la investigación, se realizó un trabajo práctico que contenía las conclusiones finales del proyecto. Este estudio permitió aportar a los docentes de las instituciones educativas del Huila elementos sustanciales en la práctica pedagógica y el fortalecimiento del proceso de formación del docente.

Por otra parte, una tendencia que se encontró en las investigaciones consultadas de la Facultad de Educación, fueron estudios vinculados al proceso de enseñanza, lo cual permitió aportar a la región del Huila y a la Universidad Surcolombiana estrategias y planes de acción que mejoraran el proceso de enseñanza de determinadas áreas del conocimiento.

La investigación de los años 1985 a 1990, fue determinante para los estudios que se realizarían en la década de los años 90 's, muchos de los trabajos realizados durante el periodo de tiempo ya mencionado, fueron el catalizador para los posteriores trabajos.

Uno de los trabajos a resaltar durante 1985 a 1990, fue el desarrollado por el Instituto Colombiano de Bienestar Familiar Regional Huila y la Universidad Surcolombiana, que buscó indagar acerca de la idiosincrasia de la población y elaborar un análisis de la información orientada a la reflexión y a la búsqueda de líneas de prevención y acción frente al problema de las Farmacodependencias.

Otro proyecto tendiente a mejorar las condiciones de vida de los habitantes del departamento del Huila y que seguía la misma línea de intervención a las comunidades, fue la investigación General de Alfabetización para el Departamento del Huila. Este proyecto se gestó a partir de las experiencias de Alfabetización realizadas por el programa de Lingüística y Literatura de la Facultad de Educación en asocio con otros programas de la misma facultad y de la Facultad de Ciencias de la Salud, además se constituyó en el espacio propicio para que la Universidad Surcolombiana realizará acciones de acercamiento a las comunidades marginadas del Departamento, para que con ellos se buscarán alternativas de solución a sus problemas socio-culturales y se impulsará el desarrollo de la región Surcolombiana.

Todas estas investigaciones y experiencias pedagógicas, dieron origen a la propuesta de Estructuración del sistema de Investigación de la Universidad Surcolombiana, el cual se proponía abordar el estudio de problemas y potencialidades del Departamento por sectores de actividad (social, económica, política, cultural, institucional demográfica, ecológica y geográfica) y los de la Universidad por aspectos administrativos y funciones (investigación, docencia y extensión). 
En la década del 90, tomando como base los documentos consultados, se puede afirmar que en la Facultad de Educación y en la Universidad no había una política de investigación dirigida al sector educativo que articulara los contextos sociales, políticos y económicos de la región.

Como problemática visible del proceso de formación docente, se hizo necesario un estudio que determinará el desarrollo del proceso enseñanza-aprendizaje de la lengua materna en los centros educativos oficiales de básica primaria de la ciudad de Neiva.

Debido a la complejidad de las implicaciones negativas y positivas que se obtuvieron de la formación docente, fue necesario elaborar un trabajo que brindara a través de la investigación y capacitación, las herramientas adecuadas al proceso de práctica docente, coherente con la realidad de las instituciones educativas. Fue así como se constituyó el proyecto Reestructuración de las Normales Superiores de Neiva y Gigante - Ciclo Complementario de Formación Docente que tuvo en cuenta cinco aspectos de formación (pedagógico, disciplinar, investigativa, comunicación y ética y valores humanos), que sirvieron como eje de aprendizaje y los incorporó a tres núcleos definidos en el proceso de estructuración curricular: identidad, construcción de Conocimiento y Comunicación. Esta investigación se desarrolló en los niveles de Pre-escolar, Básica y Media de las Normales referenciadas.

Aún más preocupante fue el informe del sistema nacional de educación, en los años 90 denominado Saber, que estableció el desarrollo de las habilidades lecto-escritoras de los estudiantes, pues "...mostró que los alumnos no habían logrado un nivel acorde con el grado de escolaridad en el que están ubicados". Así mismo investigaciones como: "La enseñanza de la lengua materna en la escuela primaria de Neiva"; "hacia el desarrollo de la lengua materna desde el aula de clase"; "el discurso y desarrollo de la competencia comunicativa en la educación básica primaria"; "perspectivas teóricas y metodológicas", y "Los libros de texto de español para la Básica Primaria", permitieron obtener un documento en el cual se identificaron las problemáticas que aquejaban el panorama educativo en grados $1^{\circ}-2^{\circ}-3^{\circ}-4^{\circ}-5^{\circ}$ de Básica primaria. Así lo expusieron las profesoras Magdalena Arias Bonilla y María de los Ángeles Rivera Ortiz en el documento "Los Maestro y los niños leen y escriben".

La iniciativa buscó fortalecer la formación de los educadores en los procesos de lectoescritura, habilidades fundamentales en el desarrollo del lenguaje, en la lectura y producción de textos significativos que enriquecieran la visión y el quehacer pedagógico de los docentes.

Otras propuestas arrojaron resultados relevantes en relación con la labor docente y la formación educativa de niños, entre las que se puede mencionar el Centro de Investigaciones y Desarrollo Científico -CIDEC, que realizó la intervención, análisis y evaluación de los procesos elaborados para determinar la relación existente entre el rendimiento académico motriz, en los niños de las escuelas Guillermo Montenegro y Sagrada Familia, de la ciudad de Neiva. En el estudio se expusieron las explicaciones, las causas del rendimiento académico y las complejas relaciones existentes entre rendimiento intelectual y rendimiento motor en los niños observados-estudiados.

En 1996 se realizó el núcleo temático de Investigación en educación física, con el fin de aportar elementos metodológicos que permitieran la estructuración de proyectos de investigación. Se elaboró una serie de talleres, que sirvieron de guía para obtener una 
visión crítica y creativa sobre el tema. También, aportó elementos metodológicos que permitieron la realización de proyectos de investigación.

Las investigaciones que produjeron módulos, talleres, guías y cartillas educativas, estaban articuladas a la preocupación por cumplir con las apuestas orientadas, que el Ministerio de Educación Nacional, a través de la Ley 115 de 1994 (Ley General de Educación), en cuanto a calidad, tecnología, acceso, creación e innovación de planes educativos articulados con la realidad.

Gran parte de los trabajos investigativos desarrollados por la Facultad de Educación en los años 90 estuvieron enfocados en la formación docente, el diseño curricular, los PEI, la planeación educativa, la enseñanza de la lengua materna y el desarrollo de las competencias comunicativas en la educación básica.

Este último tema se caracterizó por avanzar en el estudio del contexto comunicativo, formulando nuevas estrategias, actividades y ejercicios para ampliar y profundizar el desarrollo de la competencia comunicativa de los niños por medio de prácticas interactivas.

El trabajo realizado por la Facultad de Educación de la Universidad Surcolombiana en términos de compromiso social y acercamiento a las comunidades, contribuyó al hallazgo de problemáticas en el Huila y la región sur del país durante la década de los años 90; dificultades que fueron afrontadas y permitieron el advenimiento de estrategias puntuales, que permitió la creación de pregrados y postgrados que respondieran a las circunstancias y al contexto en el cual se ubicaba.

A través de las investigaciones, la Facultad de Educación de la Universidad Surcolombiana, contribuyó en el mejoramiento de la enseñanza del idioma inglés en Neiva y el Huila. El compromiso de la universidad en esta temática correspondió principalmente a apoyar el desarrollo de los planes regionales y nacionales de educación en lo relacionado con la formación de docentes de inglés en pregrado.

El aporte de la Facultad de Educación a la Universidad Surcolombiana, a la región y al país, fue importante en la medida en que desde cada una de sus disciplinas del conocimiento brindó elementos para la consolidación de procesos académicos e investigativos en la región. Además, sirvió de insumo para la construcción y consolidación de una política de investigación en la Institución.

A lo largo de los años, la política de investigación se ha estructurado desde la creación del CESCO, el CIDEC, la DIGEIN y, finalmente, la Vicerrectoría de Investigación y Proyección Social, para iniciar una propuesta en investigación con miras a establecer las funciones y directrices que regulan el sistema de investigación teniendo en cuenta los recursos financieros y los estímulos que apoyan el desarrollo de investigaciones para la producción del conocimiento científico en la región surcolombiana.

La Universidad Surcolombiana en su política de investigación se ha soportado en normas y leyes que han gestionado el Estado y entidades gubernamentales, los cuales se han referenciado en documentos legales como el Estatuto de Investigación, que inició su proceso de reestructuración en función a la cultura investigativa; este documento fue liderado por los docentes Norberto Insuasty Plaza y Enrique Peña Forero.

* Universidad Surcolombiana, Facultad de Educación. Revista Paideia No.15 Agosto de 2010. 
El Estatuto de Investigación se consideró herramienta fundamental para el avance de investigaciones; sin embargo, frente al contexto globalizado en el desarrollo de la ciencia y la tecnología requería una mayor responsabilidad social de la Universidad en consonancia con procesos investigativos en relación con la formación en investigación, a los estímulos docentes y a la generación del conocimiento en el sector productivo.

El Acuerdo 064 de 2001 se derogó y se creó un nuevo Estatuto de Investigación el Acuerdo No. 013 de 2005, que reglamentó el sistema de investigación y las actividades de investigación relacionadas con la política de investigación.

La Facultad de Educación, incentivó la producción intelectual de los docentes para la producción del conocimiento. Este momento tomó un nuevo posicionamiento en el desarrollo de la Universidad Surcolombiana con un pensamiento innovador que se orientó a proyectos relacionados con el currículo, la evaluación, el proceso de enseñanza, la formación docente, la gestión de instituciones educativas, las prácticas pedagógicas a comunidades indígenas y los factores de retención.

En el año 2000 se formalizó el convenio de Cooperación Interinstitucional entre INCORA, Regional Huila y la Universidad Surcolombiana para el acompañamiento de comunidades indígenas en el departamento del Huila con el fin de proporcionar aspectos fundamentales en la elaboración de planes de vida. Este proyecto lo lideraron los docentes Ofelia Ramírez Losada, Jesús María Vidal Arias, Jorge Abel Bermúdez y Jorge Armando Ruiz, cuyo objetivo principal fue elaborar un Plan de Vida fundamentado en los pilares básicos social, cultural, político, económico, ambiental y las relaciones sociales, que permita el ordenamiento del pensamiento y el territorio para el fortalecimiento de la comunidades indígenas.

Dentro del marco de este convenio, los docentes investigadores avanzaron en la elaboración de los planes de vida de la Comunidad Fiw Páez Nueva Irlanda, de la Comunidad Yanacona San Agustín-Huila, de la Comunidad Yanacona Palmar de Criollo-Pitalito, de la Comunidad Nasa Páez Pic Kue La Argentina - Huila, del Resguardo Nasa Guambiano y de la Comunidad Nasa -Los Ángeles (La Plata Huila).La investigación abarcó todos los aspectos sociales y culturales de la comunidad, lo que permitió identificar las debilidades y las fortalezas a partir de la formulación de los perfiles de proyectos que apuntan a la solución de necesidades.

Es importante señalar, que el desarrollo de estas investigaciones de carácter institucional contribuyó en la organización de las comunidades indígenas del departamento del Huila con el apoyo de entidades gubernamentales.

Por otra parte, en la Facultad de Educación las investigaciones se centraron en programas de formación docente, dirigidos a los educadores del departamento del Huila y del municipio de Neiva en áreas relacionadas con la educación en valores, la pedagogía y la investigación, con el fin de proporcionar elementos sustanciales en su labor docente, razón por la cual se consideró necesario en ahondar en actividades pedagógicas para el fortalecimiento del proceso de formación profesional.

La investigación Programa de Formación Pedagógica para profesionales del Departamento del Huila en ejercicio docente (sede Neiva: 2000) la lideró el docente Hipólito Camacho Coy, cuyo objetivo fue desarrollar un proceso de formación docente que propicie la problematización de su propia práctica y genere interrogantes que puedan ser 
asumidos como objeto de análisis, reflexión, investigación y reformulación de la práctica pedagógica. El programa contó con una estructura curricular que se dividió en varias áreas: investigación, pedagogía, y socio.- psicohumanística.

En el proceso de formación los docentes pretendieron profundizar y afianzar los conocimientos básicos para aportar a su práctica pedagógica. Es conveniente señalar que en esa época la evaluación de desempeño respondía a unas exigencias del magisterio para ascender en el escalafón y mejorar su remuneración, razón por la cual la Facultad de Educación realizó diferentes tipos de programa de formación que obedecían a los criterios de ascenso al escalafón.

La Universidad Surcolombiana entró en un proceso de reestructuración académica con miras al desarrollo de la modernización curricular institucional. El espíritu que inspiraba estas reformas era mejorar la formación integral del profesional en competencias y habilidades según el desarrollo social y económico de la región.

La Facultad de Educación adelantó la investigación Propuesta Curricular Integral por los docentes Nidia Guzmán Durán y Edgar Alirio Insuasty, quienes consideraron pertinente avanzar en la integración de la lengua extranjera en las áreas de estudios y los lineamientos curriculares según la Ley General de Educación (115 de 1994).

Por otra parte los docentes Hipólito Camacho Coy, Eduardo Castillo Lugo y Jaime Monje Mahecha avanzaron en la investigación Educación Física, Programas de $6^{\circ}$ a $11^{\circ}$. Una Alternativa Curricular, que buscaba exponer la programación curricular del área de Educación Física del grado sexto a decimoprimero.

La Universidad Surcolombiana, por medio de sus diferentes políticas institucionales referenciadas en sus documentos fundamentales (Proyecto Educativo Institucional, Plan de Desarrollo 2009-2012 en sus respectivos Acuerdos donde se reglamenta lo pertinente a la docencia, la investigación y la proyección social) advierte la necesidad de avanzar en un proceso de deconstrucción curricular que permita intervenir las actuales formas de selección, organización y distribución del conocimiento considerado básico en la formación de profesionales en las diferentes áreas del conocimiento.

El profesor Nelson Ernesto López Jiménez presentó a la comunidad los reultados de la investigación Modernización Curricular de la Universidad Surcolombiana que formuló una propuesta de reestructuración curricular de los programas académicos de la Universidad Surcolombiana. Dicha investigación desarrolló un análisis detenido de las estructuras curriculares que integran los programas académicos de pregrado de la Universidad Surcolombiana. Los resultados de la investigación se convirtieron en un documento de discusión y reflexión para los Grupos de Investigación y Comités Curriculares que permitieron ahondar en la pertinencia de los procesos formativos del profesional.

De igual manera, los docentes de la Facultad de Educación centraron sus investigaciones en estudios relacionados con el proceso de enseñanza en las diferentes áreas del conocimiento; estas investigaciones se orientaron a la problemática del sistema educativo de las instituciones educativas y de la educación superior.

En la década del 2000, la educación superior enfrentó una serie de exigencias que

** Proyectos Planes de Vida. Comunidad Fiw Páez Nueva Irlanda, de la Comunidad Yanacona San Agustín-Huila, de la Comunidad Yanacona Palmar de Criollo-Pitalito, de la Comunidad Nasa Páez Pic Kue La Argentina - Huila, del Resguardo Nasa Guambiano y de la Comunidad Nasa -Los Ángeles 
respondieron a la nueva normatividad, las transformaciones y cambios por los decretos y leyes que promueve el gobierno intentaron afectar la atmósfera académica de la educación superior. Los docentes Nelson Ernesto López Jiménez, Ana Victoria Puentes de Velázquez, Wisberto Navarro Salcedo, Helga Truque Guzmán y Guillermo Sánchez Amaya consideraron pertinente avanzar en una investigación que analizara las relaciones de poder y control que subyacen en las prácticas pedagógicas de las universidades del CRES CENTRO OCCIDENTE (Caldas, Risaralda, Quindío, Tolima, Huila y Caquetá).

Igualmente el profesor Nelson Ernesto López avanzó en la investigación sobre el discurso pedagógico oficial, pedagógico institucional y pedagógico específico, a través de las prácticas pedagógicas de la Universidad Surcolombiana, lo cual permitió determinar las distancias y tensiones entre el Discurso Pedagógico Oficial y las prácticas pedagógicas institucionales.

La Facultad de Educación adelantó estudios relacionados con la problemática de la evaluación en la educación superior. Como líder en esta línea de investigación el profesor Nelson Ernesto López Jiménez desarrolló dos investigaciones. Una denominada Acerca de la problemática de la evaluación de la calidad de la educación en Colombia que aportó a la construcción de un referente teórico y conceptual hacia la consolidación de la cultura de la evaluación de la calidad de educación en Colombia. La segunda investigación se convirtió en la segunda parte del estudio Estado del arte de la evaluación de la calidad de la educación en Colombia, que tuvo como propósito la integración de las diferentes producciones y elaboraciones en materia de evaluación.

Por otra parte, la Universidad Surcolombiana, la Secretaría de Educación, el Grupo de Investigación PACA y Grupo de Investigación GIPE, establecieron una alianza estratégica con el propósito de indagar de manera sistemática los factores de retención escolar en 70 establecimientos educativos del departamento del Huila. La investigación obedeció a dos fases iniciales: consolidación del referente teórico básico y elaboración de diagnósticos de los 70 establecimientos educativos.

Siguiendo la misma línea la Universidad Surcolombiana, la Corporación Universitaria del Huila CORHUILA y la Universidad Cooperativa, Seccional Neiva, avanzaron en una alianza estratégica por indagar sobre el fenómeno de retención escolar en estas tres instituciones. La investigación se orientó a la elaboración de un proceso de complementación y articulación con los diversos trabajos relacionados al abandono estudiantil.

Es importante mencionar que en la Facultad de Educación se gestaron diversas investigaciones tendientes a estudiar la problemática del sistema educativo y de la región surcolombiana que aportaron a la comunidad universitaria propuestas y planes de acción que consolidaron el sistema de investigaciones de la Universidad Surcolombiana y la Vicerrectoría de Investigación y Proyección Social.

\section{CONCLUSIONES:}

a) La mayoría de trabajos de investigación analizados en el período 1970-2010 se caracterizan por una orientación metodológica muy arraigada en la descripción y el análisis documental. El enfoque investigativo predominante responde a los requerimientos empírico-analíticos. Se evidencia un desarrollo muy incipiente en investigaciones de tipo histórico-hermenéutico y crítico social. 
b) La mayoría de trabajos realizados obedecen a iniciativas individuales que tienen un impacto local que no generan una acción sostenida en el tiempo.

c) La pertinencia de los desarrollos investigativos con las necesidades de los procesos misionales de la Universidad, no se ha garantizado en estas cuatro décadas de existencia de la Facultad de Educación, quizá obedece a la ausencia de criterios para definir las líneas de investigación que determinen la naturaleza, los alcances y los impactos de la investigación a desarrollar.

d) En relación con los impactos de los resultados de la investigación realizada por la Facultad de Educación en estas cuatro décadas, se puede afirmar, que ha contribuido a crear condiciones para el desarrollo y consolidación de la cultura de la investigación, no obstante, se insiste en la necesidad de una política institucional de la Universidad Surcolombiana que oriente y determine su horizonte de acción.

e) Se considera necesario establecer una estructura de indicadores de pertinencia investigativa, que dote de argumentos sólidos, prácticos y accesibles a los diferentes Grupos de Investigación, en la prospectiva estratégica de crear escenarios de cambio gradual de los desarrollos investigativos, muy en consonancia con el Plan de Desarrollo Institucional 2015-2024.

Finalmente, es necesario caracterizar el resultado de esta investigación, como un primer intento de sistematización (entendida como la construcción de conocimiento a partir de la experiencia) de la actividad investigativa en la Facultad de Educación, que lejos de crear estados de satisfacción y justificación, debe asumir la dinámica del reto como exigencia válida de crecimiento, desarrollo y consolidación. Un futuro estudio orientado en esta línea genealógica debe insistir en el análisis pormenorizado de las "estructuras de poder" que expliquen lo investigado y el porqué de su realización.

\section{BIBLIOGRAFIA}

- ÁLVAREZ de Alarcón Grace, PUENTES de Velásquez Ana Victoria y VIDAL Jesús María. Competencias Básicas Sentidos y Significados desde la Perspectiva de los Docentes. 2004, Universidad Surcolombiana.

- ÁLVAREZ de Alarcón Grace, PUENTES de Velásquez Ana Victoria, GUZMÁN Baena William y VIDAL Arias Jesús María. Gestión: Un Aporte para el Mejoramiento de las Instituciones Educativas. 2010, Universidad Surcolombiana.

- ARAUJO Motta Ofelia y ROJAS Nohora Elena. Anteproyecto: Estudio Etnográfico Regional sobre Fármaco Dependencias en el Departamento del Huila. 1986, Universidad Surcolombiana.

- ARDILA Amezquita José Antonio. Sentido y Uso de Lenguaje Matemático en la Escuela. 2002, Universidad Surcolombiana.

- ARIAS Bonilla Magdalena y RIVERA Ortiz María de los Ángeles. La Enseñanza de la Lengua Materna en las Escuelas de Neiva-Huila. 1991, Universidad Surcolombiana.

- AULADELL Vidal Felipe. La genealogía como método y el uso genealógico de la historia. Revista de Filosofía: http://serbal.pntic.mec.es/ cmunoz11/vidal29.pdf 
- BAHAMÓN Cerquera Pablo Emilio. La Motricidad en Colombia; Desde lo Instrumental a lo Trascendental. 2010, Universidad Surcolombiana.

- BAHAMÓN Pablo Emilio, VARGAS Eivar Fernando, CUELLAR Juan Carlos y CHAMORRO Saulo Andrés. Implementación de una Estrategia Pedagógica de Expresiones Motrices para Construir Tejido Social en Niños y Jóvenes de los Barrios Palmas II y Neiva Ya, Comuna No. 10 de la Ciudad de Neiva. 2009, Universidad Surcolombiana.

- BAIGORRIA Nélida. Genealogía Del Derrumbe Educativo. Centro de Estudios Filosóficos Eugenio Pucciarelli. Buenos Aires. 2007.

- BELTRÁN de Rojas Clara Elvira, GIRALDO Poveda Ramón Alipio, RIVERA Ortiz María de los Ángeles y VALENCIA Giraldo Juan Clímaco. Proyecto General para la Alfabetización en el Departamento del Huila. 1987, Universidad Surcolombiana.

- BERNAL de Rojas Aura Elena y LÓPEZ Jiménez Nelson Ernesto. Reestructuración de las Escuelas Normales del Departamento del Huila: Impacto y Proyección de las Normales de Neiva Gigante y Pitalito. 1996, Universidad Surcolombiana.

- BERNAL de Rojas Aura Elena, BOHÓRQUEZ Lozano Hilda Marina, GUAYARA Roa Gilma, LONDOÑO Betancourt Gustavo, LÓPEZ Jiménez Nelson Ernesto y SÁNCHEZ Guillermo. 1997, Universidad Surcolombiana.

- BERNAL de Rojas Aura Elena, CHARRY Rodrigo, MOTTA Millán Álvaro, VELÁSQUEZ Ana Victoria, QUINTERO Charry Alba Luz y RODRÍGUEZ Luis Carlos. Situación de la Docencia en la Universidad Surcolombiana II Semestre de 1983.1984. Universidad Surcolombiana.

- BOHÓRQUEZ de Valderrama Hilda M. Estado Actual de la Enseñanza de la Física en el Nivel Medio Oficial y Privado del Huila. 1983, Universidad Surcolombiana.

- BOHÓRQUEZ Lozano Hilda Marina. Propuesta de Diseño y Elaboración de un Calendario Divulgativo sobre Ciencia y Tecnología para el Departamento del Huila. 1996, Universidad Surcolombiana.

- BOHÓRQUEZ Lozano Hilda Marina. Recreo, su Significación e Impacto en el Huila. 2002, Universidad Surcolombiana.

- CAMACHO Coy Hipólito. Evaluación Experimental de la Propuesta Curricular Alternativa para la Educación Física Secundaría. 1993, Universidad Surcolombiana.

- CASTEL Robert. Presente y Genealogía del Presente. Una aproximación no evolucionista al cambio social. Disponible en: http://seminarioteroriasymetodos.pbworks.com/ f/R.+CASTEL+-+Presente+y+genealog\%C3\%ADa+del+presente.pdf

- CERÓN Edgar William. Foucault Lector de Nietzsche: A propósito de la Genealogía y la Guerra. (2009). Philpapers.

- Consejo Nacional de Educación Superior. Acuerdo por lo Superior 2034. 2014. Editorial, Carlos Mario Lopera.

- CORTES Murcia Rafael. Atlas Lingüístico. Universidad Surcolombiana, 1979. 
- CRISTANCHO, Diana Patricia, COLUNJE, Julián, MORA Disnarda, IRIARTE Antonio, BOHORQUEZ de Valderrama Hilda y YOVANOVICH Daniel. Perfil Teórico del Docente para el Departamento del Huila. 1984, Universidad Surcolombiana.

- CRISTANCHO, Diana Patricia, COLUNJE, Julián, MORA Disnarda, IRIARTE Antonio, BOHORQUEZ de Valderrama Hilda y YOVANOVICH Daniel. Perfil Teórico del Docente para el Departamento del Huila II parte. 1984, Universidad Surcolombiana.

- FAJARDO de Carvajal Margarita y LAVAO de Serrato María Ligia. La Ternura Animada. 1998, Universidad Surcolombiana.

- FOUCAULT Michel. Nietzsche, la genealogía, la historia. Disponible en: http://www. pensament.com/filoxarxa/filoxarxa/pdf/Michel\%20Foucault\%20-\%20Nietzschegenealogiahistoria.pdf

- GARCIA Trujillo Sergio. La historia y las genealogías Una lectura del texto de Foucault Nietzsche, la genealogía, la historia. Fundación Universitaria Los Libertadores. Red de Revistas Científicas de América Latina, el Caribe, España y Portugal. Colombia. (2011)

- GARCÍA Valdés Leonardo. Fundamentos para la Formación Deportiva. 2001, Universidad Surcolombiana.

- GÓMEZ Arévalo Amaral Palevi. Una genealogía de la educación en El Salvador. Revista Latinoamericana de Estudios Educativos. (México). 2011.

- GUZMÁN Durán Nidia e INSUASTY Edgar Alirio. Propuesta Curricular Integral. 2002, Universidad Surcolombiana.

- HERNÁNDEZ Francesc J. BELTRÁN José y MARRERO Adriana. Capítulo 26. De la arqueología a la genealogía de la educación. Disponible en: http://www.uv.es/fjhernan/ docencia/curs2011_2012/unimajors2011/foucault.pdf

- JIMÉNEZ Sánchez José J. Una Genealogía de los Derechos Humanos. Universidad de Granada. Revista del Instituto de Bartolomé de las Casas.

- LÓPEZ Jiménez Nelson Ernesto y PUENTES de Velásquez Ana Victoria. Acerca de la Problemática de la Evaluación de la Calidad de la Educación en Colombia. 2008, Universidad Surcolombiana.

- LÓPEZ Gil María Aracelly. Genealogía de la práctica pedagógica para la formación de maestros normalistas. 2010. DIALNET. Disponible en: http://dialnet.unirioja.es/servlet/ articulo?codigo $=3659471$

- LÓPEZ Jiménez Nelson Ernesto y PUENTES de Velásquez Ana Victoria. Resignificar la Escuela como Escenario de Cambio y Transformación. 2010, Universidad Surcolombiana.

- LÓPEZ Jiménez Nelson Ernesto, PUENTES de Velásquez Ana Victoria, OSPINA Salazar Danixa y CABALLERO Sánchez Javier. Formar en Investigación Algo más que Discurso. 2005, Universidad Surcolombiana. 
- LÓPEZ Jiménez Nelson Ernesto, PUENTES Ana Victoria, NAVARRO Wisberto, SÁNCHEZ Amaya Guillermo y TRUQUE Guzmán Helga. Análisis de las Relaciones entre el Discurso Pedagógico Oficial, Pedagógico Institucional y Pedagógico Específico a través de las Prácticas de la Universidad Colombiana. Colciencias-Usco 2000. 2004, Universidad Surcolombiana.

- LÓPEZ Jiménez Nelson Ernesto, PUENTES de Velásquez Ana Victoria y BONILLA Castillo Marcia Fernanda. El Estado del Arte de la Evaluación de la Calidad de Educación. II Parte. 2010, Universidad Surcolombiana.

- LÓPEZ Jiménez Nelson Ernesto. Discurso Pedagógico Oficial. 2002, Universidad Surcolombiana.

$\bullet$ . Modernización Curricular de la Universidad Surcolombiana: Integración e Interdisciplinariedad. 2008, Universidad Surcolombiana.

- LÓPEZ Ordoñez Sara Milena. Modulo sobre Informática Educativa y Medios Audiovisuales. 2010, Universidad Surcolombiana.

- MARTINEZ Novillo Javier Rujas. Genealogía y Discurso de Nietzsche a Foucault. Universidad Complutense de Madrid. Revista critica de ciencias sociales y jurídicas. (2010)

- MENDIETA Eduardo. Hacer Vivir y Dejar Morir: Foucault y la Genealogía del Racismo. (2000). Conferencia.

- MORALES Álvarez Justo. Análisis Crítico del Discurso del Proceso de Paz, en Colombia Durante el Gobierno del Presidente Andrés Pastrana Arango con las Farc-EP (19982002). 2003, Universidad Surcolombiana.

- MOSCARDÓ Alcañiz Mercedes. GENEALOGÍA DEL CAMBIO SOCIAL. Universitat Jaume I de Castelló. Disponible en: file:///C:/Users/Natalia\%20PACA/Downloads/GENEALOG\%C3\%8DA\%20DEL\%20CAMBIO\%20SOCIAL.pdf.

- MUÑOZ Luís Armando y YOVANOVICH Daniel. Evaluación Experimental de un Modelo Curricular de Educación Física para Preescolar. 1993, Universidad Surcolombiana.

- NARVÁEZ Zamora Luis Javier, FRANCO Ruiz Carlos Arturo y GUERRERO Vladimir Alvear. Aplicación al Modelo Didáctico de Enseñanza Aprendizaje por Investigación en el Área de Ciencias Naturales. 2008, Universidad Surcolombiana.

- NASCIMENTO Amós. Una Genealogía de la Postmodernidad en el Contexto Latinoamericano. Disponible en: http://132.248.35.1/cultura/Ponencias/PONENCIAS2005/ Amos.htm

- OROZCO Rojas Ana Orsidis y INSUASTY Plaza Norberto. Los Cambuches de Panorama: una Experiencia Poblacional de Invasores Urbanos en Neiva. 1998, Universidad Surcolombiana. 1998, Universidad Surcolombiana.

- Programa de español y Literatura. Proyecto de Reestructuración del Centro de Estudios Surcolombianos. Universidad Surcolombiana. 1978, CESCO. 
- PUENTES de Velásquez Ana Victoria. Investigación Cualitativa. Fundamentos Conceptuales y Metodológicos su Aplicación en la Educación. 2004, Universidad Surcolombiana.

- RAMÍREZ Bahamón Jairo. Política y Escuela Radical del Tolima Grande; Huila y Tolima: Sucesos Políticos, Sociales y Educativos 1861 - 1886. 1998, Universidad Surcolombiana.

- RAMÍREZ Losada Ofelia y CUENCA Wilson Amparo. Indígenas en la USCO, sin el Derecho a la Diferencia. 2010, Universidad Surcolombiana.

- RAMÍREZ Losada Ofelia y PACHÓN Maldonado Ana Lucinda. Una Forma para Entender la Ciudad "Relaciones Sociales y Prácticas Culturales en Tres Escenarios de Mercado". 2004, Universidad Surcolombiana.

- RUTI G. Teitel. Genealogía de la Justicia Transicional. Universidad de Chile. (2003).

- SÁNCHEZ Amaya, Tomás. Aproximación a un estudio genealógico de la evaluación educativa en Colombia, segunda mitad del siglo XX. Centro de Estudios Avanzados en Niñez y Juventud alianza de la Universidad de Manizales y el CINDE. Tesis. 2008

- TERÁN Oscar. Foucault: Genealogía y Microfísica del Poder. Revista Dialéctica.

- TIERRADENTRO Andrade Lázaro, ANDRADE Salazar William, CHARRY Conde Edgar, PULIDO Ortega Héctor Raúl y ALEXIS William. Perfil del Desarrollo Físico y Motor del Niño Huilense. 2000, Universidad Surcolombiana.

- TOVAR Dussan Miguel Ángel. Centro de Investigaciones y Desarrollo Científico. Cidec. 1995, Universidad Surcolombiana.

- UNESCO. Conferencia Mundial sobre la Educación Superior. Octubre 1998, Paris.

- Conferencia Mundial sobre la Educación Superior. "la nueva dinámica de la educación Superior y la investigación para el cambio y el desarrollo". Julio 2009, Paris.

. Informe Sobre la Educación Superior en América Latina y el Caribe. 2000-2005. Editorial, Metrópolis C.A. 2006.

- VALLES Miguel S. Técnicas Cualitativas de Investigación Social Reflexión metodológica y práctica profesional. Editorial Síntesis. 1999. Madrid.

- VANEGAS Rubio Luis Evelio y ZAMBRANO Castillo Liliana Cecilia. La Enseñanza del Idioma Inglés en la Educación Básica Primaria del Municipio de Neiva Zona Urbana. 1995, Universidad Surcolombiana.

- VELASCO Amaya Hermann Omar. Foucault, la genealogía, la historia..., la verdad. Universidad de Guadalajara. 2008.

- VIDAL Jesús María, RUIZ Jorge Armando, RAMÍREZ Ofelia y BERMÚDEZ Jorge Abel. Convenio de Capacitación y Asesoría a Comunidades Rurales del Huila Beneficiarias de la Ley 160 de 1994. 2000, Universidad Surcolombiana. 
versidad Surcolombiana.

Criollo-Pitalito. 2001, Universidad Surcolombiana.

Plan de Vida de Comunidad Yanacona Palmar de

- Visión Colombia II Centenario. Fundamentar el crecimiento y el desarrollo social en la ciencia, la tecnología y la innovación. Departamento Nacional de Planeación y Colciencias.

- WARBUTON Nigel. Resumen y Crítica de la Genealogía de la Moral. Disponible en: www.alcoberro.info

- ZABALA López de Goicoechea Javier. Las razones comprehensivas y la fundamentación de los Derechos Humanos. Genealogía de una ética de los DDHH. Universidad Complutense de Madrid.

- ZÚÑIGA Camacho Gilma. La Influencia del Proyecto COFE en los Programas de Lenguas de las Universidades Colombianas. 1997, Universidad Surcolombiana.

- ZÚÑIGA Escobar Mirian y LONDOÑO Luis Oscar. Estudio sobre Experiencias Significativas de Educación Popular de Adultos en Colombia. 1995, Universidad Surcolombiana. 